\title{
Avaliação de Desempenho em Bioinformática: Estudo de caso de sistemas computacionais para a investigação de microRNAs
}

\author{
Rosana R. Aguiar ${ }^{1}$, Leandro A. Ambrosio ${ }^{1}$, Gonzalo Sepúlveda-Hermosilla ${ }^{2}$, \\ Vinicius Maracaja-Coutinho ${ }^{2,3,4}$, Alexandre Rossi Paschoal ${ }^{1, *}$ \\ * paschoal@utfpr.edu.br \\ ${ }^{1}$ Federal University of Technology - Paraná, Brazil. \\ ${ }^{2}$ Centro de Genómica y Bioinformática, Universidad Mayor, Santiago, Chile. \\ ${ }^{3}$ Beagle Bioinformatics, Santiago, Chile. \\ ${ }^{4}$ Instituto Vandique, João Pessoa, Brazil.
}

\begin{abstract}
This paper describe in details the computational evaluation of the miRQuest (http://mirquest.integrativebioinformatics.me) system which is a webserver and middleware architecture integrating different standardized softwares for microRNA (miRNA) investigation.
\end{abstract}

Resumo. Este artigo apresenta em detalhes a avaliação do sistema web miRQuest (http://mirquest.integrativebioinformatics.me) que foi implementado e fez a integração dos principais preditores de microRNAs (miRNAs) em uma arquitetura de middleware, aplicado em um estudo com de caso em bioinformática.

\section{Introdução}

A bioinformática é uma área interdisciplinar a fim de auxiliar as pesquisas para analisar e interpretar a informação de valor contida na massa de dados biológicos que vem sendo gerados [ARAÚJO et al., 2005; PADILHA et al., 2008]. Na bioinformática tem-se o estudo dos RNAs não-codificadores, do inglês non-coding RNAs (ncRNAs), que são sequências de ácidos ribonucleicos transcritas, e que diferentemente dos mais conhecidos RNAs mensageiros (mRNA), não são traduzidos em proteínas, mas ainda assim possuem funções importantíssimas para a regulação e manutenção dos processos biológicos celulares [MACHADO-LIMA et al., 2008; OLIVEIRA et al., 2011].

O miRNA ou microRNA é uma classe de ncRNA que desperta maior interesse de pesquisa pela comunidade científica. miRNAs são pequenos RNAs, contendo cerca de 22 nucleotídeos (nt), responsáveis pelo controle pós-transcricionais dos níveis de RNA mensageiro nas células via o pareamento estrutural complementar entre as duas moléculas de RNAs: miRNA-mRNA [ZHANG et al., 2007]. Este interesse se deve pelo papel de controle e regulação das redes gênicas dentro das células, estando assim envolvidas em diversos processos biológicos e doenças como câncer ou em planta, por exemplo [BARROS-CARVALHO et al. 2014; SEVERINO et al., 2013].Outro exemplo é que de acordo com o banco de dados NRDR (The Non-coding RNA Databases Resource) [PASCHOAL et al., 2012], 51\% (70 de 137, versão 2.0) dos bancos indexados que são referentes as moléculas de RNAs são específicos apenas para a classe de miRNAs. 
Atualmente, se sabe que existem diversos programas para identificação de miRNA, que foram desenvolvidos utilizando as mais diferentes linguagens e métodos computacionais como: o agrupamento (cluster) ou aprendizagem de máquina [MACHADO-LIMA et al., 2008]. Entretanto, entender o funcionamento de cada um deles e aplicá-los torna-se uma tarefa complexa. Este artigo apresenta a avaliação de desempenho do middleware miRQuest um Web Server que integra quatro dos principais preditores para investigação de miRNAs.

\section{2. ncRNAs e miRNAs}

A partir dos resultados da análise de projetos de transcriptomas, se descobriu que grande parte do que era transcrito nos genomas (p.ex. camundongo e humano) eram regiões não traduzidas, ou seja, que não geravam proteínas como um produto final. Evidências apontam que o aumento na complexidade dos organismos vivos, observados ao longo do processo evolutivo, parece estar diretamente associado à quantidade e variedade de ncRNAs, indicando que quanto mais próximo dos eucariotos superiores, maior a quantidade de ncRNAs [TAFT et al., 2007]. Em 1993, Lee e colaboradores (1993) caracterizaram e descreveram uma classe específica de ncRNA definida como miRNA ou miRNA, responsáveis por regular negativamente os níveis de expressão mRNAs nas células. O banco de dados miRBase [KOZOMARA e GRIFFITHS-JONES, 2014] sendo o estado da arte em informações referente a miRNAs, é um repositório que descreve essas moléculas como grandes responsáveis por diversos processos biológicos e doenças, resultando na classe de ncRNA mais estudada [PASCHOAL et al., 2012].

\section{Trabalhos relacionados}

Em 2008, Machado-Lima e colaboradores (2008) descreveram uma extensa lista de abordagens e técnicas computacionais para pesquisa de ncRNAs e que possuem uma relação direta com os miRNA, visto que o entendimento a respeito da modelagem computacional associada à biologia de RNAs é a base para os algoritmos de identificação deste tipo molécula. Já em 2012, Allmer e Yousef (2012) comentaram sobre algumas ferramentas e abordagens para predição de miRNAs, discutindo também os aspectos e padrões utilizados para predição de miRNAs (p. ex. estrutura, sequência, tamanho). Em linha similar, Gomes e colaboradores (2013) discorrem em uma revisão sobre ferramentas computacionais para identificação miRNAs quinze abordagens e técnicas de diferentes programas de predição. Apesar do grande número de revisões bibliográficas publicadas, verificou-se a falta e necessidade de um sistema integrado de modo a facilitar o uso de preditores para identificação de miRNAs, em especial para profissionais com poucos conhecimentos de ambientes computacionais não amigáveis, como o caso dos biólogos.

\section{Materiais e metodologias}

\subsection{Busca dos preditores de miRNA}

Foi realizada nos mecanismos de busca PubMed, ACM, IEEE e Google Scholar uma investigação por programas de identificação de miRNA. Os resultados foram analisados e os seguintes preditores escolhidos: Triplet-SVM [XUE et al., 2005]; MiPred [JIANG et al., 2007]; HHMMiR [KADRI et al., 2009] e NovoMIR [TEUNE e STEGE, 2010].

\subsection{Conjunto de dados e experimentos para avaliação de desempenho}

Foram usados 1872 sequencias miRNAs de Homo sapiens e 298 de Arabidopsis thaliana do miRBase versão 19. Na avaliação de desempenho, primeiramente foi 
analisado o tempo de execução. Dados com sequências de distintos tamanhos foram usados. Para humano usou-se 10, 50, 100, 500 e 1.000 sequências e planta de 10, 30, 50, 100 e 200 sequências. O segundo experimento foi de performance. As sequências de ambos genomas foram usadas como conjunto de dados positivo (os dados de treinamento foram retirados). Já o conjunto de dados negativo foi extraído de um estudo publicado por Janssen e colaboradores (2008), que contém sequências de proteínas e um grupo de sequências randômicas aleatórias que sabidamente não correspondem miRNAs. Para medir o desempenho usou-se as seguintes métricas: sensibilidade; sensibilidade; precisão; acurácia e F1-Score (Powers, 2011).

\subsection{Desenvolvimento do miRQuest}

O miRQuest foi desenvolvido como aplicação web na linguagem Java com o emprego do conceito em camadas utilizando o Tomcat versão 8 como Web Server. O XML é usado como formato padrão para comunicação entre as etapas de processamento do middleware e Shell Scripts, em linguagem PERL, foram desenvolvidos para execução de etapas específicas como RNAfold [BRAMEIER e WIUF, 2007] e RNAshapes [STEFFEN et al., 2006]. RNAfold e RNAshapes são programas de predição de estrutura secundária, uma etapa usada por alguns preditores para identificação de características referentes à estrutura dos miRNAs identificados.

O miRQuest foi também implementado usando Contexts and Dependency Injection (CDI) para integração das camadas, Apache Shiro Framework para administração de seção e Commons Email API para envio de email. Cada ferramenta de predição foi instalada conforme as regras descritas em suas documentações. E ainda, para a execução das ferramentas foram aplicados os parâmetros e padrões conforme recomendado no manual de uso de cada ferramenta.

\section{Resultados e discussão da avaliação do miRQuest}

As tabelas 1 e 2 apresentam os resultados para humano e a tabela 3 para planta. Analisando-se os dados destas tabelas observou-se a diferença no tempo de execução quando comparamos a execução stand-alone com a execução via web.

Tabela 1. Resultado do teste de tempo dos programas Triplet-SVM (A) e HHMMiR (B) com 10, 50, 100, 500 e 1.000 sequências em humano. Em azul o tempo executando comandos stand-alone; vermelho via web com miRQuest.

\begin{tabular}{|c|c|c|c|c|c|c|c|c|c|c|}
\hline $\mathrm{a}$ & \multicolumn{2}{|c|}{10} & \multicolumn{2}{|c|}{50} & \multicolumn{2}{|c|}{100} & \multicolumn{2}{|c|}{500} & \multicolumn{2}{|c|}{1000} \\
\hline b & \multicolumn{2}{|c|}{$1,1 \mathrm{~KB}$} & \multicolumn{2}{|c|}{$5,4 \mathrm{~KB}$} & \multicolumn{2}{|c|}{$10,7 \mathrm{~KB}$} & \multicolumn{2}{|c|}{$54,6 \mathrm{~KB}$} & \multicolumn{2}{|c|}{$107,5 \mathrm{~KB}$} \\
\hline & $\mathrm{A}$ & B & $\mathrm{A}$ & B & A & $\mathrm{B}$ & $\mathrm{A}$ & $\mathrm{B}$ & $\mathrm{A}$ & $\mathrm{B}$ \\
\hline 1 & $0 \mathrm{~m} 0.051 \mathrm{~s}$ & $0 \mathrm{~m} 0.053 \mathrm{~s}$ & $0 \mathrm{~m} 0.183 \mathrm{~s}$ & $0 \mathrm{~m} 0.193 \mathrm{~s}$ & $0 \mathrm{~m} 0.343 \mathrm{~s}$ & $0 \mathrm{~m} 0.360 \mathrm{~s}$ & $0 \mathrm{~m} 1.849 \mathrm{~s}$ & $0 \mathrm{~m} 1.962 \mathrm{~s}$ & $0 \mathrm{~m} 3.689 \mathrm{~s}$ & $0 \mathrm{~m} 3.692 \mathrm{~s}$ \\
\hline 2 & $0 \mathrm{~m} 0.049 \mathrm{~s}$ & $0 \mathrm{~m} 0.092 \mathrm{~s}$ & $0 \mathrm{~m} 0.086 \mathrm{~s}$ & $0 \mathrm{~m} 0.107 \mathrm{~s}$ & $0 \mathrm{~m} 0.132 \mathrm{~s}$ & $0 \mathrm{~m} 0.139 \mathrm{~s}$ & $0 \mathrm{~m} 0.510 \mathrm{~s}$ & $0 \mathrm{~m} 0.094 \mathrm{~s}$ & $0 \mathrm{~m} 0.939 \mathrm{~s}$ & $0 \mathrm{~m} 0.205 \mathrm{~s}$ \\
\hline 3 & $0 \mathrm{~m} 0.011 \mathrm{~s}$ & $0 \mathrm{~m} 0.092 \mathrm{~s}$ & $0 \mathrm{~m} 0.015 \mathrm{~s}$ & $0 \mathrm{~m} 0.144 \mathrm{~s}$ & $0 \mathrm{~m} 0.016 \mathrm{~s}$ & $0 \mathrm{~m} 0.422 \mathrm{~s}$ & $0 \mathrm{~m} 0.036 \mathrm{~s}$ & $0 \mathrm{~m} 0.908 \mathrm{~s}$ & $0 \mathrm{~m} 0.046 \mathrm{~s}$ & $0 \mathrm{~m} 1.754 \mathrm{~s}$ \\
\hline & $0 \mathrm{~m} 0.111 \mathrm{~s}$ & $0 \mathrm{~m} 0.237 \mathrm{~s}$ & $0 \mathrm{~m} 0.284 \mathrm{~s}$ & $0 \mathrm{~m} 0.444 \mathrm{~s}$ & $0 \mathrm{~m} 0.491 \mathrm{~s}$ & $0 \mathrm{~m} 0.921 \mathrm{~s}$ & $0 \mathrm{~m} 2.395 \mathrm{~s}$ & $0 \mathrm{~m} 2.964 \mathrm{~s}$ & $0 \mathrm{~m} 4.674 \mathrm{~s}$ & $0 \mathrm{~m} 5.651 \mathrm{~s}$ \\
\hline & $0 \mathrm{~m} 3.00 \mathrm{~s}$ & $0 \mathrm{~m} 2.00 \mathrm{~s}$ & $0 \mathrm{~m} 4.00 \mathrm{~s}$ & $0 \mathrm{~m} 3.00 \mathrm{~s}$ & $0 \mathrm{~m} 4.00 \mathrm{~s}$ & $0 \mathrm{~m} 4.00 \mathrm{~s}$ & $0 \mathrm{m5.00s}$ & $0 \mathrm{~m} 5.00 \mathrm{~s}$ & $0 \mathrm{~m} 10.00 \mathrm{~s}$ & $0 \mathrm{~m} 9.00 \mathrm{~s}$ \\
\hline
\end{tabular}

Fonte: o autor.

Notas. $^{\text {a e b }}$ significam respectivamente tamanho da sequência e tamanho do arquivo. E os números 1,2 e 3 correspondem ao conjunto de comandos que cada ferramenta possui para o processamento das sequencias. Na execução stand-alone cada comando precisa ser processado separadamente, o resultado em azul corresponde a soma desses tempos. 
Tabela 2. Resultado do teste de tempo do preditor MiPred.

\begin{tabular}{lccccc}
\hline $\mathrm{a}$ & \multicolumn{1}{c}{50} & 100 & 500 & 1000 \\
\cline { 2 - 6 } $\mathrm{b}$ & $1,1 \mathrm{~KB}$ & $5,4 \mathrm{~KB}$ & $10,7 \mathrm{~KB}$ & $54,6 \mathrm{~KB}$ & $107,5 \mathrm{~KB}$ \\
\cline { 2 - 6 } Local & $1 \mathrm{~m} 44.183 \mathrm{~s}$ & $11 \mathrm{~m} 17.601 \mathrm{~s}$ & $25 \mathrm{~m} 48.489 \mathrm{~s}$ & $148 \mathrm{~m} 30.318 \mathrm{~s}$ & $278 \mathrm{~m} 11.054 \mathrm{~s}$ \\
Web & $2 \mathrm{~m} 33.00 \mathrm{~s}$ & $15 \mathrm{~m} 5.00 \mathrm{~s}$ & $27 \mathrm{~m} 47 \mathrm{~s}$ & $189 \mathrm{~m} 25.00 \mathrm{~s}$ & $329 \mathrm{~m} 04.00 \mathrm{~s}$ \\
\hline Fonte: o autor. \\
Nota.
\end{tabular}

O sistema miRQuest expressou um tempo maior de execução devido a integração dos Preditores - que é um custo normal necessário. E, nos testes stand-alone, não foi avaliado o tempo que o usuário leva para escrever os comandos, foi considerado apenas a execução real dos comandos. Outro aspecto perceptível é que o MiPred (Tabela 2) é a ferramenta que mais demanda tempo de execução. HHMMiR (Tabela 1) é a ferramenta mais rápida para execução, em geral, quando comparada ao Triplet- SVM e ao NovoMIR (Tabela 1 e 3 , respectivamente).

Tabela 3. Teste de tempo utilizando organismo de planta na ferramenta NovoMIR com 10, 30, 50, 100 e 200 sequências.

\begin{tabular}{lccccc}
\hline $\mathrm{a}$ & \multicolumn{1}{c}{30} & 50 & 100 & 200 \\
\cline { 2 - 6 } $\mathrm{b}$ & $2,2 \mathrm{~KB}$ & $5,5 \mathrm{~KB}$ & $8,8 \mathrm{~KB}$ & $19,3 \mathrm{~KB}$ & $42,6 \mathrm{~KB}$ \\
\cline { 2 - 6 } Local & $0 \mathrm{~m} 2.203 \mathrm{~s}$ & $0 \mathrm{~m} 4.328 \mathrm{~s}$ & $0 \mathrm{~m} 7.338 \mathrm{~s}$ & $0 \mathrm{~m} .18 .539 \mathrm{~s}$ & $0 \mathrm{~m} .44 .789 \mathrm{~s}$ \\
$\mathrm{Web}$ & $0 \mathrm{~m} .3 .00 \mathrm{~s}$ & $0 \mathrm{~m} .7 .00 \mathrm{~s}$ & $0 \mathrm{~m} 10.00 \mathrm{~s}$ & $0 \mathrm{~m} 24.00 \mathrm{~s}$ & $0 \mathrm{~m} 56.00 \mathrm{~s}$ \\
\hline
\end{tabular}

Fonte: o autor.

Nota. $^{\text {a e b }}$ tem o mesmo significado da tabela 1 .

$\mathrm{Na}$ análise de performance - comparação entre as ferramentas (benchmarking) os resultados demonstram que o NovoMIR é o melhor preditor para plantas e o MiPred para humano, conforme dados da Tabela 4. A surpresa foram os resultados obtidos para o preditor HHMMiR, uma vez que essa ferramenta foi desenvolvida e treinada, principalmente, para predição de miRNAs em humanos.

Tabela 4. Resultado do benchmarking dos preditores. Testes feitos em humano e planta utilizando o sistema miRQuest. Em verde está destacado as melhores pontuações.

\begin{tabular}{llllll}
\hline & Triplet-SVM & MiPred & HHMMiR & NovoMIR & \\
\hline \multirow{3}{*}{ A.thaliana } & $64,09 \%$ & $34,90 \%$ & $9,06 \%$ & $77,27 \%$ & Sensibilidade \\
& $87,47 \%$ & $96,48 \%$ & $91,91 \%$ & $99,50 \%$ & Acurácia \\
& $16,99 \%$ & $51,49 \%$ & $6,32 \%$ & $90,27 \%$ & Precisão \\
& $26,86 \%$ & $41,60 \%$ & $7,45 \%$ & $83,27 \%$ & F1-Score \\
\hline \multirow{3}{*}{ H. sapiens } & $59,42 \%$ & $92,81 \%$ & $0,66 \%$ & $40,28 \%$ & Sensibilidade \\
& $83,20 \%$ & $97,72 \%$ & $79,95 \%$ & $88,56 \%$ & Acurácia \\
& $52,42 \%$ & $94,18 \%$ & $2,44 \%$ & $98,56 \%$ & Precisão \\
Ambos & $55,70 \%$ & $93,49 \%$ & $1,04 \%$ & $57,19 \%$ & F1-Score \\
\hline
\end{tabular}




\section{Conclusão}

Os resultados apresentaram um tempo maior para execução via web, mas o miRQuest, mostrou-se viável por não apresentar grande discrepância de tempo com relação a execução stand-alone. No que se refere ao benchmarking, realizado pelo miRQuest, demonstrou-se os bons resultados para o NOVOMIR e o MiPred. Tem-se interesse futuro de adicionar outros preditores ao miRQuest e testar o seu desempenho em arquitetura Cloud Computing.

\section{Agradecimentos}

ARP agradece pelo suporte do projeto ao CNPq - Edital MCTI/CNPQ/Universal 14/2014 - Faixa A - até R\$ 30.000,00 Processo: 454505/2014-0. Esta pesquisa é parte do resultado de mestrado da aluna RRA do Programa de Pós-Graduação em Informática (Profissional) UTFPR Cornélio Procópio, PR, Brasil.

\section{Referências}

Allmer J1, Yousef M. Computational methods for ab initio detection of microRNAs. Front Genet. 2012 Oct 10; 3:209. doi: 10.3389/fgene.2012.00209. eCollection 2012.

Araújo DAM, Maracaja-Coutinho V, Padilha IQM, Rego TG. (2005) Genômica e Bioinformática: importância e perspectivas para o Nordeste. Ciência e Cotidiano. 1:5-9.

Barros-Carvalho GA, Paschoal AR, Marcelino-Guimarães FC, Hungria M. (2014) Prediction of potential novel microRNAs in soybean when in symbiosis. Genet Mol Res. 13(4):8519-29. doi: 10.4238/2014.October.20.28.

Brameier M, Wiuf C (2007). Ab initio identification of human microRNAs based on structure motifs. BMC Bioinformatics, 8:478.

Gomes CP1, Cho JH, Hood L, Franco OL, Pereira RW, Wang K. (2013) A Review of Computational Tools in microRNA Discovery. Front Genet. 15; 4:81. doi: 10.3389/fgene.2013.00081.

Janssen S. et. al. (2008). Shape based indexing for faster search of RNA family databases. BMC Bioinformatics, 9:131.

Jiang P. et. al. (2007). MiPred: classification of real and pseudo microRNA precursors using random forest prediction model with combined features. Nucleic Acids, 35:339-344.

Kadri S. et al. (2009) HHMMiR: efficient de novo prediction of microRNAs using hierarchical hidden Markov models. BMC Bioinformatics. 10 Suppl 1:S35.

Kozomara A. and Griffiths-Jones S. (2014) miRBase: annotating high confidence microRNAs using deep sequencing data. NAR 42: D68-D73

Lee RC, Feinbaum RL, Ambros V; Feinbaum; Ambros (1993). "The C. elegans heterochronic gene lin-4 encodes small RNAs with antisense complementarity to lin-14". Cell 75 (5): 843-54. Doi: 10.1016/0092-8674(93)90529Y. PMID 8252621.

Machado-Lima, A.; del Portillo, H.A. ; DURHAM, A.M. (2008) Computational methods in noncoding RNA research. Journal of Mathematical Biology, v. 56, p. 15-49.

Oliveira KC, Carvalho MLP, Maracaja-Coutinho V, Kitajima JP, Verjovski-Almeida S (2011). Non-coding RNAs in schistosomes: an unexplored world. Anais da Academia Brasileira de Ciências, 83(2): 673-694.

Padilha IQM, Durbano JPM, Martins AB, Almeida RS, Maracaja-Coutinho V, Araújo DAM. (2008) A bioinformática como instrumento de inserção digital e difusão da biotecnologia. Revista Eletrônica Extensão Cidadã, 5 .

Paschoal AR, Maracaja-Coutinho V, Setubal JC, Simões ZL, Verjovski-Almeida S, Durham AM. (2012) Non-coding transcription characterization and annotation: A guide and web resource for non-coding RNA databases. RNA BIOL, 9:274-282.

Powers, D.M.W. (2011) Evaluation: FromPrecision, Recall and F-Measureto ROC, Informedness, Markedness e Correlation. Journal of Machine Learning Technologies, 2 (1) 37-63.

Severino P, Oliveira LS, Torres N, Andreghetto FM, Klingbeil Mde F, Moyses R, Wünsch-Filho V, Nunes FD, Mathor MB, Paschoal AR, Durham AM. (2013) High-throughput sequencing of small RNA transcriptomes reveals critical biological features targeted by microRNAs in cell models used for squamous cell cancer research. BMC Genomics. 14:735. doi: 10.1186/1471-2164-14-735.

Steffen P. et al. (2006) RNAshapes: an integrated RNA analysis package based on abstract shapes. Bioinformatics. 22(4):500-3.

Taft RJ, Pheasant M, Mattick JS. (2007). The relationship between non-protein-coding DNA and eukaryotic complexity. Bioessays. 29(3):288-99.

Teune J.-H. and Steger, G. (2010) NOVOMIR: De Novo Prediction of MicroRNA-Coding Regions in a Single PlantGenome. Journal of Nucleic Acids.

Xue C. et al. (2005) Classification of real and pseudo microRNA precursors using local structure-sequence features and support vector machine. BMC Bioinformatics, 6:310.

Zhang, B. et al. (2007) MicroRNAs and their regulatory roles in animals and plants. J. Cell. Physiol., 210:279-289. 